\section{Apoio social e prática de atividade física em adolescentes da rede pública de ensino: qual a importância da família e dos amigos?}

\author{
Social support and physical activity in adolescents \\ from public schools: the importance of family \\ and friends
}

\author{
Apoyo social y práctica de actividad física en \\ adolescentes de la escuela pública: ¿Cuál es \\ la importancia de la familia y los amigos?
}

\begin{abstract}
The aim of this study was to verify the association between different types and sources of social support and physical activity among adolescents from Curitiba, Paraná State, Brazil. A schoolbased survey was conducted with a representative sample of adolescents from public schools ( $n=1,469)$. Multiple regression models were used to test the association between weekly frequency and sources of social support from family and friends and weekly frequency of physical activity. Among boys, frequent company of family (PR: 2.88; 95\%CI: 2.00-4.13) and friends (PR: 5.46; 95\%CI: 2.33-12.78) and positive reinforcement from friends (PR: 1.81; 95\%CI: 1.18-2.77) were positively associated with physical activity. Sporadic invitation by the family was negatively associated with physical activity (PR: 0.66; 95\%CI: 0.46-1.14). For girls, frequent company of family (PR: 3.39; 95\%CI: 1.49-7.69) and friends (PR: 4.06; 95\%CI: 2.22-7.45) increased the likelihood of physical activity. Company of friends was the most important type of social support for physical activity among these adolescents.
\end{abstract}

Social Support; Motor Activity; Students; Adolescent
Crisley Vanessa Prado 1,2

Alex Vieira Lima ${ }^{1}$

Rogério César Fermino 2

Ciro Romelio Rodriguez Añez 3

Rodrigo Siqueira Reis 1

\section{Resumo}

O objetivo deste estudo foi analisar a associação entre diferentes tipos e fontes de apoio social com a prática de atividade física exercida de adolescentes de Curitiba, Paraná, Brasil. Um inquérito de base escolar foi realizado com amostra representativa de adolescentes da rede pública de ensino $(n=1.469)$. Modelos de regressão múltipla foram utilizados para testar a associação entre a frequência semanal de apoio social recebido da família e dos amigos com a prática de atividade física. Entre os meninos, a companhia frequente da família (RP: 2,88; IC95\%: 2,00-4,13) e a dos amigos (RP: 5,46; IC95\%: 2,33-12,78), além do reforço positivo destes (RP: 1,81; IC95\%: 1,18-2,77), apresentaram associação positiva com a atividade física. O convite esporádico da família apresentou associação negativa (RP: 0,66; IC95\%: 0,46-1,14). Para as meninas, a companhia frequente da família (RP: 3,39; IC95\%: 1,49-7,69) e a dos amigos (RP: 4,06; IC95\%: 2,22-7,45) aumentam a probabilidade da realização de atividade física. Conclui-se que a companhia dos amigos é o tipo de apoio social mais importante para a prática de atividade física de adolescentes.

Apoio Social; Atividade Motora; Estudantes; Adolescente 


\section{Introdução}

A inatividade física é um dos principais fatores de risco para as doenças crônicas não transmissíveis, sendo responsável por uma a cada dez mortes no mundo 1 . Um estilo de vida ativo iniciado ainda na adolescência pode aumentar a probabilidade de prática regular de atividade física na vida adulta 2. Portanto, o estímulo à prática de atividade física na adolescência pode contribuir para o aumento dos níveis de atividade física em várias fases da vida $3,4,5$. Apesar desses benefícios, aproximadamente oito em cada dez adolescentes não atendem as recomendações mínimas de prática de atividade física 6 .

Diversos fatores estão associados com a prática de atividade física em adolescentes 7,8, dentre eles o apoio social. Este construto constitui uma das funções mais importantes das relações sociais e pode ser definido como a ajuda ou assistência recebida por meio das relações sociais e interpessoais 2,9 . No contexto da atividade física, o apoio social apresenta características relativas à participação, discussão de oportunidades relacionadas com as atividades, por meio das redes sociais formadas pelo indivíduo 10,11 . Nesse sentido, a literatura aponta duas categorias relevantes de apoio social, aquela ligada a aspectos tangíveis (concretos e observados) ou aquela associada aos aspectos intangíveis (sentimentos, valores ou percepções) 10 . A primeira inclui aquisição de equipamentos, pagamento de transportes e mensalidades para a prática de atividade física, participação ativa dos pais ou amigos e participação assistida nas atividades. A segunda envolve encorajamento, elogio, reforço positivo, informações, ou incentivo para a realização de atividade física 10 .

Ainda que a literatura tenha reportado a importância do apoio social para a prática de atividade física de adolescentes, essa associação tem sido pouco explorada em países em desenvolvimento ${ }^{6}$. No Brasil, apenas um estudo analisou essa relação entre adolescentes, e os resultados mostraram associação positiva entre a companhia dos amigos e da família com a prática de atividade física em pelo menos um dia na semana ${ }^{12}$. Até o momento, não existem estudos que tenham analisado os diferentes tipos de apoio social e sua relação com a prática de atividade física em adolescentes brasileiros, nem se essa associação difere entre os sexos. No entanto, evidências apontam que o apoio social tangível é mais comum entre os meninos, enquanto as meninas recebem maior apoio informativo 11,13,14.

Compreender a relevância dos diferentes tipos de apoio social para a atividade física pode contribuir para o delineamento de intervenções efetivas para a promoção de atividade física de adolescentes. Outra possível contribuição é reconhecer em que medida essa associação varia entre meninos e meninas. Portanto, o objetivo deste estudo foi verificar a associação entre os diferentes tipos e fontes de apoio social com a prática de atividade física de adolescentes de Curitiba, Paraná.

\section{Materiais e métodos}

Os dados do presente estudo foram extraídos do projeto Determinantes da Atividade Física e Obesidade em Escolares do Ensino Médio da Rede Pública da Cidade de Curitiba-PR, Brasil. O estudo, de base escolar, apresentou um delineamento transversal e foi realizado entre os meses de março a maio de 2006, com amostra representativa de alunos do Ensino Médio da rede pública de ensino de Curitiba.

\section{Participantes}

De acordo com a Secretaria de Educação do Estado do Paraná (SEED) 15, no ano de 2006, a população de estudantes do período diurno era de 42.563 adolescentes. Para a estimativa do cálculo amostral, foi considerado erro de $3 \%$, prevalência estimada de $50 \%$ (inatividade física), efeito de delineamento de 1,5 , excesso de $10 \%$ para a taxa de não resposta e intervalo de $95 \%$ de confiança. Assim, a amostra necessária foi calculada em 1.609 escolares 12 .

A amostra foi selecionada em dois estágios. No primeiro, 14 escolas foram aleatoriamente selecionadas a partir de uma listagem fornecida pela SEED. A proporcionalidade de alunos foi estabelecida de acordo com o número de matrículas em cada uma das nove regionais da cidade e o número de alunos em cada uma das séries do ensino médio. Em seguida, 62 turmas foram selecionadas e visitadas. Adolescentes com idade superior a 18 anos, aqueles portadores de necessidades especiais ou incapazes de responder as questões foram excluídos da amostra. Os que não apresentaram o termo de consentimento livre e esclarecido assinado pelo responsável foram considerados como perdas. No total, 1.618 adolescentes foram entrevistados.

\section{Coleta de dados}

A coleta de dados foi realizada de maneira coordenada em sala de aula, com questionário padronizado, por dois entrevistadores previamente treinados. $\mathrm{O}$ estudo foi aprovado pelo comitê de 
ética em pesquisa da Pontifícia Universidade Católica do Paraná (CEP-PUCPR; no 1076/2006), e os procedimentos de coleta de dados seguiram as recomendações do Sistema Nacional de Ética em Pesquisa.

\section{Variável dependente}

\section{- Prática de atividade física}

A prática de atividade física foi avaliada por meio de uma questão utilizada em inquéritos populacionais 16 , na qual os adolescentes reportaram em quantos dias, ao longo de uma semana habitual, realizavam atividade física de intensidade moderada a vigorosa (AFMV), com duração maior ou igual a sessenta minutos por dia 17 . Os adolescentes foram classificados em "inativos/ insuficientemente ativos" ( $\leq 4 \mathrm{dias} / \mathrm{sem}$ AFMV, $\geq 60 \mathrm{~min}$ ) e "fisicamente ativos" ( $\geq 5 \mathrm{dias} / \mathrm{sem}$ AFMV, $\geq 60 \mathrm{~min}$ ), de acordo com as recomendações atuais de prática de atividade física nessa faixa etária 18.

\section{Variável independente}

\section{- Apoio social}

O apoio social para a prática de atividade física é um construto multidimensional, caracterizado por ações provenientes de diferentes fontes e pessoas, como, por exemplo, a família e os amigos. Pode variar em diferentes tipos: levar, convidar, acompanhar, incentivar e elogiar o jovem para a realização de atividades físicas, como também acompanhá-lo e elogiá-lo nessas ações 10.

No presente estudo, o apoio social foi avaliado com cinco questões relacionadas com a frequência semanal com que a família ou os amigos realizavam ações para facilitar ou incentivar a realização de atividade física ${ }^{19}$. Cada questão apresentou cinco opções de resposta (nunca, uma vez, às vezes, quase todos os dias, todos os dias). Para efeitos de análise, as opções "uma vez" e "às vezes", bem como as opções "quase todos os dias" e "todos os dias", foram agrupadas para compor as categorias de apoio social "às vezes" e "sempre", respectivamente.

\section{Covariáveis}

Na revisão da literatura, foram identificadas algumas variáveis potencialmente associadas com o apoio social e a prática de atividade física em adolescentes: faixa etária, índice de massa corporal (IMC), nível socioeconômico e escolaridade do adulto responsável pela maior renda do domicílio 8,10,20,21. No presente estudo, essas variáveis foram avaliadas, selecionadas e testadas como possíveis covariáveis.

\section{- Faixa etária}

A faixa etária foi avaliada por meio de uma questão relacionada com a idade dos adolescentes, os quais posteriormente foram classificados em cinco faixas etárias: 14, 15, 16, 17 e 18 anos.

- IMC

O IMC dos adolescentes foi calculado com base nas medidas de massa corporal e estatura autorreportadas 22. Para a classificação do IMC, utilizaram-se os pontos de corte recomendados para o uso de comparações internacionais 23. Os jovens foram classificados com IMC "normal” (baixo peso e peso normal) e "sobrepeso" (excesso de peso e/ou obesidade).

\section{- Nível socioeconômico}

O nível socioeconômico foi classificado de acordo com a posse de utensílios domésticos e os adolescentes classificados em cinco classes econômicas ${ }^{24}$. Para fins de análise os indivíduos foram agrupados em três níveis: $\mathrm{A}(\mathrm{A} 1+\mathrm{A} 2$ - maior renda), $\mathrm{B}(\mathrm{B} 1+\mathrm{B} 2)$ e $\mathrm{C}(\mathrm{C}+\mathrm{D}+\mathrm{E})$.

\section{- Escolaridade do adulto responsável pela renda do domicílio}

A escolaridade foi avaliada com uma questão sobre o grau de instrução pessoal e agrupada em quatro categorias de ensino: Fundamental incompleto, Fundamental completo, Médio completo e Superior completo 24 .

\section{Análise dos dados}

Dos 1.618 adolescentes entrevistados, 149 não apresentavam dados completos sobre as variáveis utilizadas no presente estudo, tendo sido excluídos das análises. Restaram 1.469 participantes, sendo esta a amostra analítica para esta investigação.

Os dados foram analisados distribuindo-se a frequência absoluta e a relativa, para comparar as proporções de prática de atividade física entre as categorias de apoio social. A regressão de Poisson foi utilizada para analisar a associação entre apoio social e prática de atividade física. Inicialmente, foi realizado o variance inflation factor test e rejeitou-se a hipótese de multicolinearidade. Após a elaboração do modelo bruto, todas as variáveis independentes e as covariáveis foram consideradas para a elaboração do modelo de 
regressão multivariável inicial, mantendo-se no modelo multivariável final apenas as seguintes covariáveis: nível socioeconômico, idade, IMC, escolaridade dos pais e sexo, juntamente com as demais variáveis de apoio social. As variáveis entraram no mesmo nível de análise pelo método de entrada forçada. Foi testada, ainda, a interação entre sexo, apoio social e prática de atividade física, considerando-se o sexo masculino como a categoria de referência. As análises foram realizadas no Stata 11 (Stata Corp., College Station, Estados Unidos) e levaram em consideração o procedimento adotado na seleção da amostra, recorrendo-se ao comando "svy" do software. As análises foram estratificadas por sexo, e o nível de significância, mantido em $5 \%$.

\section{Resultados}

Participaram deste estudo 1.469 adolescentes, sendo a maior parte do sexo feminino (59,2\%); a taxa de recusa foi inferior a $5 \%(n=79)$. A maior proporção dos indivíduos pertencia à faixa etária de 16 anos $(32,9 \%)$, apresentava IMC normal (88,3\%) e nível socioeconômico B $(63,1 \%)$; os pais tinham Ensino Médio completo $(35,1 \%$ ) (Tabela 1). A prática de atividade física em pelo menos cinco dias na semana foi de $14,5 \%$, sendo os meninos os mais ativos ( $\mathrm{p}<0,001)$.

$\mathrm{O}$ apoio social mais comum recebido da família foi o reforço positivo $(45,1 \%$; "dizer que acha bom”), enquanto o menos reportado foi a participação dos familiares durante a realização

Tabela 1

Características descritivas dos participantes de acordo com o sexo. Curitiba, Paraná, Brasil, 2006.

\begin{tabular}{|c|c|c|c|c|c|c|c|}
\hline \multirow[t]{2}{*}{ Variável } & \multicolumn{2}{|c|}{ Meninos $(n=600)$} & \multicolumn{2}{|c|}{ Meninas $(n=869)$} & \multirow[t]{2}{*}{ Valor de $p$} & \multicolumn{2}{|c|}{ Total $(\mathrm{N}=1.469)$} \\
\hline & $\mathbf{n}$ & $\%$ & $\mathbf{n}$ & $\%$ & & $\mathbf{n}$ & $\%$ \\
\hline \multicolumn{8}{|l|}{ Participantes } \\
\hline \multicolumn{8}{|l|}{ Faixa etária (anos) } \\
\hline 14 & 28 & 4,7 & 56 & 5,7 & 0,023 & 84 & 5,7 \\
\hline 15 & 143 & 23,8 & 370 & 25,2 & & 370 & 25,2 \\
\hline 16 & 194 & 32,3 & 290 & 33,4 & & 484 & 32,9 \\
\hline 17 & 180 & 30,0 & 230 & 26,5 & & 410 & 27,9 \\
\hline 18 & 55 & 9,2 & 66 & 7,6 & & 121 & 8,2 \\
\hline \multicolumn{8}{|l|}{ IMC } \\
\hline Normal & 503 & 83,8 & 794 & 91,4 & $<0,001$ & 1.297 & 88,3 \\
\hline Sobrepeso & 97 & 16,2 & 75 & 8,6 & & 172 & 11,7 \\
\hline \multicolumn{8}{|l|}{ Nível socioeconômico } \\
\hline C & 94 & 15,7 & 174 & 20,0 & 0,006 & 268 & 18,2 \\
\hline B & 378 & 63,0 & 549 & 63,2 & & 927 & 63,1 \\
\hline A (maior renda) & 128 & 21,3 & 146 & 16,8 & & 274 & 18,7 \\
\hline \multicolumn{8}{|l|}{ Escolaridade do pai (ensino) } \\
\hline Fundamental incompleto & 107 & 17,8 & 223 & 25,7 & 0,001 & 330 & 22,5 \\
\hline Fundamental completo & 124 & 20,7 & 190 & 21,9 & & 314 & 21,4 \\
\hline Médio completo & 233 & 38,8 & 282 & 32,5 & & 515 & 35,1 \\
\hline Superior completo & 136 & 22,7 & 174 & 20,0 & & 310 & 21,1 \\
\hline \multicolumn{8}{|l|}{ Prática de AFMV (dias/semana) } \\
\hline$\leq 4$ & 467 & 77,8 & 789 & 90,8 & $<0,001$ & 1.256 & 85,5 \\
\hline$\geq 5$ & 133 & 22,2 & 80 & 9,2 & & 213 & 14,5 \\
\hline \multicolumn{8}{|l|}{ Apoio social da família } \\
\hline \multicolumn{8}{|c|}{ Levaram para praticar atividade física } \\
\hline Nunca & 342 & 57,0 & 538 & 61,9 & 0,366 & 880 & 59,9 \\
\hline Às vezes & 224 & 37,3 & 265 & 30,5 & & 489 & 33,3 \\
\hline Sempre & 34 & 5,7 & 66 & 7,6 & & 100 & 6,8 \\
\hline
\end{tabular}

(continua) 
Tabela 1 (continuação)

\begin{tabular}{|c|c|c|c|c|c|c|c|}
\hline \multirow[t]{2}{*}{ Variável } & \multicolumn{2}{|c|}{ Meninos $(n=600)$} & \multicolumn{2}{|c|}{ Meninas $(n=869)$} & \multirow[t]{2}{*}{ Valor de $p$} & \multicolumn{2}{|c|}{ Total $(\mathrm{N}=1.469)$} \\
\hline & $\mathrm{n}$ & $\%$ & $\mathrm{n}$ & $\%$ & & $\mathrm{n}$ & $\%$ \\
\hline \multicolumn{8}{|c|}{ Apoio social da família } \\
\hline \multicolumn{8}{|c|}{ Convidaram para praticar atividade } \\
\hline \multicolumn{8}{|l|}{ física } \\
\hline Nunca & 264 & 44,0 & 336 & 38,7 & 0,066 & 600 & 40,8 \\
\hline Às vezes & 277 & 46,2 & 439 & 50,5 & & 716 & 48,7 \\
\hline Sempre & 59 & 9,8 & 94 & 10,8 & & 153 & 10,4 \\
\hline \multicolumn{8}{|c|}{ Praticaram atividade física juntos } \\
\hline Nunca & 283 & 47,2 & 417 & 48,0 & 0,759 & 700 & 47,7 \\
\hline Às vezes & 278 & 46,3 & 397 & 45,7 & & 675 & 45,9 \\
\hline Sempre & 39 & 6,5 & 55 & 6,3 & & 94 & 6,4 \\
\hline \multicolumn{8}{|c|}{ Incentivaram a praticar atividade física } \\
\hline Nunca & 127 & 21,2 & 166 & 19,1 & 0,574 & 293 & 20,0 \\
\hline Às vezes & 294 & 49,1 & 444 & 51,2 & & 738 & 50,3 \\
\hline Sempre & 178 & 29,7 & 258 & 29,7 & & 436 & 29,7 \\
\hline \multicolumn{8}{|c|}{ Disseram que acham bom que } \\
\hline \multicolumn{8}{|c|}{ pratique atividade física } \\
\hline Nunca & 94 & 15,7 & 107 & 12,3 & 0,779 & 201 & 13,7 \\
\hline Às vezes & 227 & 37,8 & 378 & 43,5 & & 605 & 41,2 \\
\hline Sempre & 279 & 46,5 & 384 & 44,2 & & 663 & 45,1 \\
\hline \multicolumn{8}{|c|}{ Apoio social dos amigos } \\
\hline \multicolumn{8}{|c|}{ Convidaram para praticar atividade } \\
\hline \multicolumn{8}{|c|}{ física } \\
\hline Nunca & 116 & 19,3 & 297 & 34,2 & $<0,001$ & 413 & 28,1 \\
\hline Às vezes & 293 & 48,8 & 467 & 53,7 & & 760 & 51,7 \\
\hline Sempre & 191 & 31,8 & 105 & 12,1 & & 296 & 20,1 \\
\hline \multicolumn{8}{|c|}{ Praticaram atividade física juntos } \\
\hline Nunca & 109 & 18,2 & 312 & 35,9 & $<0,001$ & 421 & 28,7 \\
\hline Às vezes & 304 & 50,7 & 444 & 51,1 & & 748 & 50,9 \\
\hline Sempre & 187 & 31,2 & 113 & 13,0 & & 300 & 20,4 \\
\hline \multicolumn{8}{|c|}{ Incentivaram a praticar atividade física } \\
\hline Nunca & 158 & 26,3 & 289 & 33,3 & $<0,001$ & 447 & 30,4 \\
\hline Às vezes & 312 & 52,0 & 462 & 53,2 & & 774 & 52,7 \\
\hline Sempre & 130 & 21,7 & 118 & 13,6 & & 248 & 16,9 \\
\hline \multicolumn{8}{|c|}{$\begin{array}{l}\text { Disseram que gostam de praticar } \\
\text { atividade física }\end{array}$} \\
\hline Nunca & 127 & 21,2 & 252 & 29,0 & $<0,001$ & 379 & 25,8 \\
\hline Às vezes & 287 & 47,8 & 439 & 50,5 & & 726 & 49,4 \\
\hline Sempre & 186 & 31,0 & 178 & 20,5 & & 364 & 24,8 \\
\hline \multicolumn{8}{|c|}{$\begin{array}{l}\text { Disseram que acham bom que } \\
\text { pratique atividade física }\end{array}$} \\
\hline Nunca & 231 & 38,5 & 308 & 35,4 & 0,712 & 539 & 36,7 \\
\hline Às vezes & 228 & 38,0 & 396 & 45,6 & & 624 & 42,5 \\
\hline Sempre & 141 & 23,5 & 165 & 19,0 & & 306 & 20,8 \\
\hline
\end{tabular}

AFMV: atividade física de intensidade moderada a vigorosa; IMC: índice de massa corporal.

das atividades $(6,4 \%)$. Ambos os tipos de apoio social não apresentaram diferença entre os sexos. Em relação ao apoio social recebido dos amigos, o fato de os amigos "dizerem que gostam de praticar atividade física” foi o mais frequente
(24,8\%), enquanto o incentivo para a realização das atividades foi o menos reportado $(16,9 \%)$, sendo ambos mais frequentemente recebidos pelos meninos $(\mathrm{p}<0,001)$ (Tabela 1$)$. 
Na análise bivariada (Tabela 2), apenas o reforço positivo da família ("disseram que acham bom que pratique atividade física”) não apresentou associação com a prática de atividade física, tanto entre meninos, quanto entre meninas. $\mathrm{Na}$ análise multivariável, apenas a companhia frequente dos familiares durante a prática de atividade física permaneceu positivamente associada com a atividade física de meninos (RP: 2,88; IC95\%: 2,00-4,13) e meninas (RP: 3,39; IC95\%: 1,49-7,69). No entanto, o convite esporádico dos familiares foi inversamente associado com a prática de atividade física entre meninos (RP: 0,66; IC95\%: 0,46-0,95).
Em relação ao apoio social recebido de amigos, os resultados da análise bivariada mostraram que todos os tipos de apoio social apresentaram associação com a prática de atividade física em meninos e meninas. Na análise multivariável apenas a companhia frequente dos amigos permaneceu associada com a atividade física para ambos os sexos (meninos RP: 5,46; IC95\%: 2,3312,78; meninas RP: 4,06; IC95\%: 2,22-7,45). O reforço positivo frequente dos amigos ("disseram que acham bom que pratique atividade física”) aumentou em $81 \%$ a probabilidade de os meninos praticarem atividade física.

\begin{tabular}{|c|c|c|c|c|c|c|}
\hline & \multicolumn{6}{|c|}{ Meninos $(n=600)$} \\
\hline & \multicolumn{4}{|c|}{ Análise bivariada } & \multicolumn{2}{|c|}{ Análise multivariável } \\
\hline & $\mathrm{n}$ & $\%$ & RP & IC95\% & $\mathbf{R P}$ & IC95\% \\
\hline \multicolumn{7}{|c|}{ Apoio social da família } \\
\hline \multicolumn{7}{|c|}{ Levaram para praticar atividade física } \\
\hline Nunca & 70 & 20,5 & 1,00 & $0,011 * \star$ & 1,00 & $0,276 * *$ \\
\hline Às vezes & 49 & 21,9 & 1,07 & $0,74-1,52$ & 1,00 & $0,68-1,49$ \\
\hline Sempre & 14 & 41,2 & 2,01 & $1,20-3,35$ & 1,35 & $0,78-2,36$ \\
\hline \multicolumn{7}{|c|}{ Convidaram para praticar atividade física } \\
\hline Nunca & 58 & 22,0 & 1,00 & 0,014 ** & 1,00 & 0,207 ** \\
\hline Às vezes & 52 & 18,8 & 0,85 & $0,58-1,25$ & 0,66 & $0,46-0,95$ \\
\hline Sempre & 23 & 39,0 & 1,77 & $1,14-2,74$ & 0,73 & $0,46-1,14$ \\
\hline \multicolumn{7}{|c|}{ Praticaram atividade física juntos } \\
\hline Nunca & 55 & 19,4 & 1,00 & $<0,001 * *$ & 1,00 & $<0,001 * *$ \\
\hline Às vezes & 54 & 19,4 & 1,00 & $0,66-1,51$ & 1,03 & $0,72-1,48$ \\
\hline Sempre & 24 & 61,5 & 3,17 & $2,15-4,65$ & 2,88 & $2,00-4,13$ *** \\
\hline \multicolumn{7}{|c|}{ Incentivaram a praticar atividade física } \\
\hline Nunca & 18 & 14,2 & 1,00 & $0,017 * *$ & 1,00 & $0,288 * \star$ \\
\hline Às vezes & 62 & 21,1 & 1,49 & $0,98-2,25$ & 1,44 & $0,96-2,16$ \\
\hline Sempre & 53 & 29,8 & 2,10 & $1,15-3,83$ & 1,46 & $0,73-2,90$ \\
\hline \multicolumn{7}{|c|}{ Disseram que acham bom que pratique atividade física } \\
\hline Nunca & 14 & 14,9 & 1,00 & $0,109 * \star$ & 1,00 & $0,222 * \star$ \\
\hline Às vezes & 41 & 18,1 & 1,21 & $0,61-2,42$ & 1,15 & $0,59-2,25$ \\
\hline Sempre & 78 & 28,0 & 1,88 & $0,85-4,14$ & 1,55 & $0,72-3,31$ \\
\hline \multicolumn{7}{|c|}{ Apoio social dos amigos } \\
\hline \multicolumn{7}{|c|}{ Convidaram para praticar atividade física } \\
\hline Nunca & 19 & 16,4 & 1,00 & $<0,001 \star \star \star$ & 1,00 & 0,076 ** \\
\hline Às vezes & 44 & 15,0 & 0,92 & $0,52-1,62$ & 0,56 & $0,28-1,14$ \\
\hline Sempre & 70 & 36,6 & 2,24 & $1,60-3,13$ & 0,55 & $0,29-1,04$ \\
\hline \multicolumn{7}{|c|}{ Praticaram atividade física juntos } \\
\hline Nunca & 11 & 10,1 & 1,00 & $<0,001 \star \star$ & 1,00 & $<0,001 \star \star$ \\
\hline Às vezes & 38 & 12,5 & 1,24 & $0,67-2,27$ & 1,69 & $0,76-3,80$ \\
\hline Sempre & 84 & 44,9 & 4,45 & $2,60-7,60$ & 5,46 & $2,33-12,78 \#$ \\
\hline
\end{tabular}

(continua) 


\begin{tabular}{|c|c|c|c|c|c|c|}
\hline & \multicolumn{6}{|c|}{ Meninos $(n=600)$} \\
\hline & \multicolumn{4}{|c|}{ Análise bivariada } & \multicolumn{2}{|c|}{ Análise multivariável } \\
\hline & $\mathrm{n}$ & $\%$ & RP & IC95\% & RP & IC95\% \\
\hline \multicolumn{7}{|c|}{ Apoio social dos amigos } \\
\hline \multicolumn{7}{|c|}{ Convidaram para praticar atividade física } \\
\hline Nunca & 19 & 16,4 & 1,00 & $<0,001 \star \star$ & 1,00 & 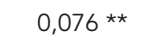 \\
\hline Às vezes & 44 & 15,0 & 0,92 & $0,52-1,62$ & 0,56 & $0,28-1,14$ \\
\hline Sempre & 70 & 36,6 & 2,24 & $1,60-3,13$ & 0,55 & $0,29-1,04$ \\
\hline \multicolumn{7}{|c|}{ Praticaram atividade física juntos } \\
\hline Nunca & 11 & 10,1 & 1,00 & $<0,001 \star \star$ & 1,00 & $<0,001 * \star$ \\
\hline Às vezes & 38 & 12,5 & 1,24 & $0,67-2,27$ & 1,69 & $0,76-3,80$ \\
\hline Sempre & 84 & 44,9 & 4,45 & $2,60-7,60$ & 5,46 & $2,33-12,78$ \\
\hline \multicolumn{7}{|c|}{ Incentivaram a praticar atividade física } \\
\hline Nunca & 22 & 13,9 & 1,00 & $<0,001 * \star$ & 1,00 & 0,587 ** \\
\hline Às vezes & 59 & 18,9 & 1,36 & $0,82-2,25$ & 0,86 & $0,49-1,49$ \\
\hline Sempre & 52 & 40,0 & 2,87 & $1,85-4,46$ & 0,88 & $0,41-1,88$ \\
\hline \multicolumn{7}{|c|}{ Disseram que gostam de praticar atividade física } \\
\hline Nunca & 15 & 11,8 & 1,00 & $<0,001 \star \star$ & 1,00 & $0,422 \star \star$ \\
\hline Às vezes & 48 & 16,7 & 1,42 & $0,83-2,42$ & 1,06 & $0,58-1,98$ \\
\hline Sempre & 70 & 37,6 & 3,19 & $1,92-5,28$ & 1,31 & $0,68-2,50$ \\
\hline \multicolumn{7}{|c|}{ Disseram que acham bom que pratique atividade física } \\
\hline Nunca & 31 & 13,4 & 1,00 & $<0,001 * \star$ & 1,00 & $0,021 * *$ \\
\hline Às vezes & 47 & 20,6 & 1,54 & $1,01-2,33$ & 1,45 & $0,86-2,46$ \\
\hline Sempre & 55 & 39,0 & 2,91 & $2,04-4,13$ & 1,81 & $1,18-2,77 \star \star \star$ \\
\hline Total & 133 & 22,2 & & & & \\
\hline
\end{tabular}

\begin{tabular}{|c|c|c|c|c|c|c|}
\hline & \multicolumn{6}{|c|}{ Meninas $(n=869)$} \\
\hline & \multicolumn{4}{|c|}{ Análise bivariada } & \multicolumn{2}{|c|}{ Análise multivariável } \\
\hline & $\mathrm{n}$ & $\%$ & $\mathrm{RP}$ & IC95\% & $\mathrm{RP}$ & $1 \mathrm{C9} 95 \%$ \\
\hline \multicolumn{7}{|c|}{ Apoio social da família } \\
\hline \multicolumn{7}{|c|}{ Levaram para praticar atividade física } \\
\hline Nunca & 35 & 6,5 & 1,00 & $<0,001 \star \star$ & 1,00 & $0,227 * \star$ \\
\hline Às vezes & 28 & 10,6 & 1,62 & $1,21-2,16$ *夫 & 1,36 & $0,89-2,10$ \\
\hline Sempre & 17 & 25,8 & 3,96 & $2,11-7,41$ & 1,37 & $0,76-2,45$ \\
\hline \multicolumn{7}{|c|}{ Convidaram para praticar atividade física } \\
\hline Nunca & 25 & 7,4 & 1,00 & $<0,001 * \star$ & 1,00 & 0,377 ** \\
\hline Às vezes & 30 & 6,8 & 0,92 & $0,49-1,72$ & 0,66 & $0,29-1,52$ \\
\hline Sempre & 25 & 26,6 & 3,57 & $2,19-5,83$ & 1,44 & $0,64-3,23$ \\
\hline \multicolumn{7}{|c|}{ Praticaram atividade física juntos } \\
\hline Nunca & 26 & 6,2 & 1,00 & $<0,001 * *$ & 1,00 & 0,007 ** \\
\hline Às vezes & 34 & 8,6 & 1,37 & $0,78-2,40$ & 1,31 & $0,59-2,91$ \\
\hline Sempre & 20 & 36,4 & 5,83 & $3,41-9,96$ & 3,39 & $1,49-7,69 \# \#$ \\
\hline \multicolumn{7}{|c|}{ Incentivaram a praticar atividade física } \\
\hline Nunca & 12 & 7,2 & 1,00 & $<0,001 \star \star$ & 1,00 & 0,725 ** \\
\hline Às vezes & 32 & 7,2 & 1,00 & $0,61-1,63$ & 0,93 & $0,69-1,25$ \\
\hline Sempre & 36 & 14,0 & 1,93 & $1,44-2,58$ & 0,86 & $0,37-1,98$ \\
\hline \multicolumn{7}{|c|}{ Disseram que acham bom que pratique atividade física } \\
\hline Nunca & 8 & 7,5 & 1,00 & 0,094 *夫 & 1,00 & 0,872 ** \\
\hline Às vezes & 24 & 6,3 & 0,85 & $0,38-1,87$ & 0,85 & $0,35-2,03$ \\
\hline Sempre & 48 & 12,5 & 1,67 & $0,90-3,09$ & 1,08 & $0,40-2,95$ \\
\hline
\end{tabular}

(continua) 


\begin{tabular}{|c|c|c|c|c|c|c|}
\hline & \multicolumn{6}{|c|}{ Meninas $(n=869)$} \\
\hline & \multicolumn{4}{|c|}{ Análise bivariada } & \multicolumn{2}{|c|}{ Análise multivariável ** } \\
\hline & $\mathrm{n}$ & $\%$ & RP & IC95\% & RP & $1 C 95 \%$ \\
\hline \multicolumn{7}{|c|}{ Apoio social dos amigos } \\
\hline \multicolumn{7}{|c|}{ Convidaram para praticar atividade física } \\
\hline Nunca & 22 & 7,4 & 1,00 & $0,012 * \star$ & 1,00 & 0,383 ** \\
\hline Às vezes & 38 & 8,1 & 1,09 & $0,66-1,82$ & 0,83 & $0,48-1,41$ \\
\hline Sempre & 20 & 19,0 & 2,57 & $1,27-5,18$ & 0,69 & $0,32-1,51$ \\
\hline \multicolumn{7}{|c|}{ Praticaram atividade física juntos } \\
\hline Nunca & 19 & 6,1 & 1,00 & $<0,001 * \star$ & 1,00 & $<0,001 * *$ \\
\hline Às vezes & 31 & 7,0 & 1,15 & $0,68-1,93$ & 1,35 & $0,68-2,68$ \\
\hline Sempre & 30 & 26,5 & 4,36 & $2,51-7,56$ & 4,06 & $2,22-7,45$ \#\#\# \\
\hline \multicolumn{7}{|c|}{ Incentivaram a praticar atividade física } \\
\hline Nunca & 21 & 7,3 & 1,00 & $<0,001 \star \star$ & 1,00 & $0,162 \star \star$ \\
\hline Às vezes & 32 & 6,9 & 0,95 & $0,64-1,42$ & 1,02 & $0,58-1,78$ \\
\hline Sempre & 27 & 22,9 & 3,15 & $1,89-5,24$ & 2,08 & $0,67-6,51$ \\
\hline \multicolumn{7}{|c|}{ Disseram que gostam de praticar atividade física } \\
\hline Nunca & 21 & 8,3 & 1,00 & $0,004 * *$ & 1,00 & 0,994 ** \\
\hline Às vezes & 30 & 6,8 & 0,82 & $0,57-1,18$ & 0,72 & $0,37-1,40$ \\
\hline Sempre & 29 & 16,3 & 1,95 & $1,04-3,69$ & 1,05 & $0,36-3,13$ \\
\hline \multicolumn{7}{|c|}{ Disseram que acham bom que pratique atividade física } \\
\hline Nunca & 27 & 8,8 & 1,00 & $0,017 * *$ & 1,00 & $0,301 * \star$ \\
\hline Às vezes & 27 & 6,8 & 0,78 & $0,52-1,16$ & 0,69 & $0,45-1,07$ \\
\hline Sempre & 26 & 15,8 & 1,80 & $1,13-2,86$ & 0,73 & $0,39-1,35$ \\
\hline Total & 80 & 9,2 & & & & \\
\hline
\end{tabular}

IC95\%: intervalo de 95\% de confiança; RP: razão de prevalência.

* Cinco ou mais dias/semana, atividade física de intensidade moderada a vigorosa $\geq 60$ minutos/dia;

** Valor de $p$ de tendência;

*** Ajustado para as demais variáveis de apoio social e as covariáveis nível socioeconômico e índice de massa corporal (IMC) ( $\mathrm{p}<0,20$ );

\# Ajustado para as demais variaveis de apoio social e covariáveis nível socioeconômico, IMC e escolaridade do responsável financeiro ( $p<0,20$ );

\#\# Ajustado para as demais variáveis de apoio social e as covariáveis faixa etária e nível socioeconômico $(p<0,20)$;

\#\#\# Ajustado para as demais variáveis de apoio social e as covariáveis faixa etária, nível socioeconômico, IMC e escolaridade do responsável

financeiro $(p<0,20)$.

Na análise de interação, as meninas que reportaram receber frequentemente o convite da família para a prática de atividade física apresentaram $91 \%$ maior probabilidade de praticar atividade física, quando comparadas aos meninos. As demais interações não foram significativas.

\section{Discussão}

Este estudo é o primeiro que analisou a associação entre os diferentes tipos e fontes de apoio social com a prática de atividade física em uma amostra representativa de adolescentes brasileiros. O apoio social da família e dos amigos é relatado como uma das estratégias possíveis para aumentar a adesão em programas de atividade física nessa faixa etária 25,26 . Na presente pesqui- sa, foram observadas diferenças nas magnitudes de associação entre tipos e fontes de apoio social com a prática de atividade física em ambos os sexos.

A companhia da família e dos amigos durante a prática de atividade física foi o tipo de apoio social mais importante para meninos e meninas. Entretanto, outro estudo 12 não encontrou associação entre as variáveis. Essa inconsistência de resultados pode ser explicada pela dicotomização do apoio social, que pode ter sido muito ampla para distinguir diferentes taxas de atividade física. Os resultados desta investigação são corroborados por outra evidência que reporta associação positiva entre falta de companhia com a inatividade física de adolescentes ${ }^{27}$. Acredita-se que a companhia e a motivação da família e dos amigos para a realização de atividade física pos- 
sibilitem maiores interações sociais, as quais podem favorecer o engajamento dos adolescentes em atividade física. Quando pessoas mais próximas estão presentes e valorizam a mudança de comportamento, os jovens elevam sua autoestima e sentem-se mais seguros e confiantes 28 . A teoria social cognitiva indica diferentes maneiras de o apoio social se relacionar com as crenças pessoais, concebendo-o como uma fonte de autoeficácia em diversos momentos e transições da vida 28 .

O convite esporádico da família para a prática de atividade física associou-se com menor probabilidade de os meninos serem fisicamente ativos. Não foram identificados estudos que avaliaram separadamente o convite para a prática de atividade física como forma de apoio social, dificultando possíveis comparações com os resultados do presente estudo. No entanto, é possível que a preferência por diferentes tipos de atividade física praticados por adultos e adolescentes possam, parcialmente, explicar esse achado. Por exemplo, enquanto as atividades físicas mais praticadas pelos adultos são a caminhada, futebol e a musculação ${ }^{29}$, entre os jovens as atividades mais comuns são o futebol, voleibol e caçador ${ }^{30}$. Portanto, uma aparente discordância quanto à atividade preferida pode ser observada, de modo que possivelmente os pais convidem os filhos para praticarem atividade física (por exemplo, caminhada) não necessariamente de preferência dos jovens. Ademais, o convite dos amigos foi mais frequente do que o recebido da família (Tabela 1), o que parece reforçar tal discordância. Cabe ressaltar que a adolescência é marcada pelo conflito por independência, fase em que os jovens procuram tomar suas próprias decisões, contrariando muitas vezes seus pais 27 . Esse aspecto é mais comum entre os meninos, por apresentarem maior liberdade do que as meninas, as quais tendem a permanecer mais tempo envolvidas em tarefas domésticas 13 .

O reforço positivo dos amigos ("disseram que acham bom que pratique atividade física") foi associado com a prática de atividade física entre os meninos. Esse achado é consistente com o de outros estudos, que demonstram que os meninos apresentam maior socialização e liberdade para realizar atividade física com os amigos 8,31. Mais uma vez, trata-se de um comportamento próprio da adolescência, que tende a ser um período de autoafirmação, autonomia e independência, quando os jovens atribuem maior relevância aos hábitos e comportamentos de seus amigos, ignorando os conselhos e atitudes de seus pais 32 .

O presente estudo avaliou o apoio social proveniente de duas fontes: família e amigos. O envolvimento desses dois grupos foi similar entre os sexos, e a companhia é o tipo de apoio social mais importante para ambos. A opinião dos amigos parece ser relevante para os meninos. Dessa forma, os adolescentes que têm companhia da família e dos amigos apresentam, respectivamente, cinco e quatro vezes maior probabilidade de realizarem atividade física em relação aos que nunca recebem esse tipo de apoio social. Tais resultados são semelhantes aos de outro estudo, que identificou que a participação em atividade física com os amigos aumentou em três e duas vezes a probabilidade de, respectivamente, meninos e meninas serem mais ativos ${ }^{31}$. Apesar de a participação da família ter sido importante para a prática de atividade física de adolescentes, o apoio dos amigos apresenta maior relevância. Um recente estudo de revisão demonstrou que o apoio social dos pais não esteve associado com a prática de atividade física de adolescentes em países de baixa e média renda 6 . De fato, com o decorrer da idade, a participação do jovem em atividade física com a família tende a diminuir, contrariamente à participação com os amigos, que aumenta 31,32 .

Os tipos e fontes de apoio social foram recentemente empregados em pesquisas para identificar suas contribuições na prática de atividade física entre adolescentes 10,14,31. Em recente estudo de revisão ${ }^{10}$, no qual foram identificadas duas dimensões de apoio social, observou-se que os tipos de apoio tangíveis são considerados um dos meios mais eficazes para o aumento no engajamento em atividade física. $\mathrm{O}$ envolvimento e a supervisão dos pais nas atividades, fornecimento de transporte, pagamento de mensalidades e a compra de equipamentos para o exercício físico facilitam a participação dos adolescentes nessa prática, além de proporcionar maior aproximação entre pais e filhos, estando estes, muitas vezes, carentes desse apoio 7 .

Apesar de a companhia da família e dos amigos para a prática de atividade física dos adolescentes ter sido mais importante neste estudo, vale ressaltar a relevância de outros tipos de apoio provenientes dessas duas fontes. Estudos relatam que o incentivo, encorajamento, apoio emocional, bem como o apoio informativo e o elogio, estão associados a maiores níveis de atividade física de adolescentes 10,20,21.

Algumas limitações devem ser consideradas ao interpretar os resultados. O delineamento do estudo não permite estabelecer a relação causal entre as variáveis, ou seja, não é possível identificar se o apoio social é de fato um fator determinante para a prática de atividade física. A prática de atividade física foi mensurada de maneira global, considerando a realização de períodos elevados de exercício físico ( $\geq 60$ minutos/ 
dia), o que pode ter subestimado os níveis dessa atividade e ainda não permitiu estabelecer relações com atividades físicas específicas. $\mathrm{O}$ apoio social da família também foi avaliado de maneira global, impossibilitando a identificação da fonte primária do apoio (pai, mãe, irmãos etc.), o que não permite verificar se esse apoio difere entre os membros da família. A faixa etária dos adolescentes limitou-se entre 14 e 18 anos de idade, o que não permite a extrapolação dos resultados para outras idades. Por fim, foram avaliados somente adolescentes de escolas públicas, impedindo a generalização dos resultados para estudantes de escolas privadas. Ainda assim, a representatividade amostral permite a extrapolação dos resultados para todos os alunos da rede pública de ensino da cidade.

\section{Conclusão}

A companhia frequente da família e dos amigos associou-se positivamente com a prática de atividade física em ambos os sexos, assim como o reforço positivo dos amigos associou-se com a prática de atividade física entre os meninos. O convite esporádico da família foi inversamente associado com a atividade física no sexo masculino. As intervenções para aumentar os níveis de atividade física na faixa etária em estudo devem priorizar a participação da família e dos amigos durante a realização das atividades, além de possibilitar maior interação entre os jovens para favorecer o reforço positivo dos amigos. Estudos de intervenção e prospectivos devem testar a influência dos diferentes tipos e fontes de apoio social nos níveis de atividade física de adolescentes, sobretudo em países de menor renda. Por fim, é necessário que outras fontes de apoio social (como pai, mãe, irmãos, professores, médico) sejam exploradas para melhor elucidar a associação entre as variáveis.

\section{Resumen}

El objetivo de este estudio fue analizar la asociación entre diferentes tipos y fuentes de apoyo social con la práctica de actividad física en adolescentes de Curitiba, Paraná, Brasil. Se realizó una encuesta de base escolar en una muestra representativa de adolescentes de la red pública de educación $(n=1.469)$. Se usaron modelos de regresión múltiple para probar la asociación entre la frecuencia semanal del apoyo social recibido de la familia y de los amigos con la práctica de actividad física. Para los jóvenes, la compañía frecuente de la familia (RP: 2,88; IC95\%: 2,00-4,13) y de los amigos (RP: 5,46; IC95\%: 2,33-12,78), presentó una asociación positiva con la actividad física. La invitación esporádica de la familia presentó una asociación negativa (RP: 0,66; IC95\%: 0,46-1,14). Para las jóvenes, la compañía frecuente de la familia (RP: 3,39; IC95\%: 1,49-7,69) y de los amigos (RP: 4,06; IC95\%: 2,22-7,45) aumentan la probabilidad de realizar actividad física. Se concluye que la compañía de los amigos es el tipo de apoyo social más importante para la práctica de actividad física en adolescentes.

Apoyo Social; Actividad Motora; Estudiantes; Adolescente 


\section{Colaboradores}

C. V. Prado participou da concepção do estudo, revisão da literatura, análise dos dados e redação inicial do manuscrito. A. V. Lima e R. C. Fermino participaram da concepção inicial do estudo, análise de dados, revisão da literatura e revisão do manuscrito. C. R. R. Añez e R. S. Reis participaram da revisão crítica do manuscrito em todas as suas etapas.

\section{Referências}

1. Lee IM, Shiroma EJ, Lobelo F, Puska P, Blair SN, Katzmarzyk PT. Effect of physical inactivity on major non-communicable diseases worldwide: an analysis of burden of disease and life expectancy. Lancet 2012; 380:219-29.

2. Azevedo MR, Araújo CL, Silva MC, Hallal PC. Tracking of physical activity from adolescence to adulthood: a population-based study. Rev Saúde Pública 2007; 41:69-75.

3. Tammelin T, Nayha S, Hills AP, Jarvelin MR. Adolescent participation in sports and adult physical activity. Am J Prev Med 2003; 24:22-8.

4. Gordon-Larsen P, Nelson MC, Popkin BM. Longitudinal physical activity and sedentary behavior trends: adolescence to adulthood. Am J Prev Med 2004; 27:277-83.

5. Oliveira AJ, Lopes CS, Ponce de Leon AC, Rostila M, Griep RH, Werneck GL, et al. Social support and leisure-time physical activity: longitudinal evidence from the Brazilian Pró-Saúde cohort study. Int J Behav Nutr Phys Act 2011; 77:1-10.

6. Bauman A, Reis RS, Sallis JF, Wells JC, Loos RJF, Martin BW. Correlates of physical activity: why are some people physically active and others not? Lancet 2012; 6736:31-44.

7. Sallis JF, Prochaska JJ, Taylor WC. A review of correlates of physical activity of children and adolescents. Med Sci Sports Exerc 2000; 32:963-75.
8. van Der Horst K, Chin APM, Twisk JWR, van Mechelen W. A brief review on correlates of physical activity and sedentariness in youth. Med Sci Sports Exerc 2007; 39:1241-50.

9. Glanz K, Rimer BK, Viswanath K. Health behavior and health education: theory, research, and practice. 2nd Ed. San Francisco: Jossey-Bass; 2008.

10. Beets MW, Cardinal BJ, Alderman BL. Parental social support and the physical activity-related behaviors of youth: a review. Health Educ Behav 2010; 37:621-44.

11. Matillas MM, Ortega FB, Ruiz JR, Gómez DM, Marcos A, Moliner-Urdiales D, et al. Adolescent's physical activity levels and relatives physical activity engagement and encouragement: the HELENA study. Eur J Public Health 2010; 21:705-12.

12. Fermino RC, Rech CR, Hino AAF, Añez CRR, Reis RS. Atividade física e fatores associados em adolescentes do ensino médio de Curitiba, Brasil. Rev Saúde Pública 2010; 44:986-95.

13. Gonçalves H, Hallal PC, Amorim TC, Araújo CLP, Menezes AMB. Fatores socioculturais e nível de atividade física no início da adolescência. Rev Panam Salud Pública 2007; 22:246-53.

14. Wright MS, Wilson DK, Griffin S, Evans A. A qualitative study of parental modeling and social support for physical activity in underserved adolescents. Health Educ Res 2010; 25:224-32. 
15. Secretaria de Educação do Estado do Paraná. Relatório estatístico de alunos matriculados. http:// wwwe.pr.gov.br/escolas/numeros (acessado em Ago/2012).

16. Brener ND, Kann L, Kinchen SA, Grunbaum JA, Whalen L, Eaton D, et al. Methodology of the youth risk behavior surveillance system. MMWR Recomm Rep 2004; 53:1-11.

17. Prochaska JJ, Sallis JF, Long B. A physical activity screening measure for use with adolescents in primary care. Arch Pediatr Adolesc Med 2001; 155:554-9.

18. Strong WB, Malina RM, Blimkie CJ, Daniels SR, Dishman RK, Gutin B, et al. Evidence based physical activity for school youth. J Pediatr 2005; 146:732-7.

19. Reis RS, Sallis JF. Validade e reprodutibilidade da versão brasileira da escala de suporte social para o exercício em adolescentes. Rev Bras Ciênc Mov 2005; 13:7-14.

20. de Vet E, de Ridder DT, de Wit JB. Environmental correlates of physical activity and dietary behaviours among young people: a systematic review of reviews. Obes Rev 2011; 12:130-42.

21. Ferreira I, van der Horst K, Wendel-Vos W, Kremers S, van Lenthe FJ, Brug J. Environmental correlates of physical activity in youth: a review and update. Obes Rev 2006; 8:129-54.

22. Farias Junior JC. Validade das medidas auto-referidas de peso e estatura para o diagnóstico do estado nutricional de adolescentes. Rev Bras Saúde Matern Infant 2007; 7:167-74.

23. Cole TJ, Bellizzi MC, Flegal KM, Dietz WH. Establishing a standard definition for child overweight and obesity worldwide: international survey. BM] 2000; 320:1240-3.
24. Associação Brasileira de Empresas de Pesquisa. Brazilian criteria for economic classification. http://www.abep.org/novo/Content.aspx? SectionID=84 (acessado em Ago/2012).

25. Salvy SJ, Roemmich JN, Bowker JC, Romero ND, Stadler PJ, Epstein LH. Effect of peers and friends on youth physical activity and motivation to be physically active. J Pediatr Psychol 2009; 34:217-25.

26. McDavid L, Cox AE, Amorose AJ. The relative roles of physical education teachers and parents in adolescents leisure-time physical activity motivation and behavior. Psychol Sport Exerc 2012; 13:99-107.

27. Santos MS, Hino AAF, Reis RS, Añez CRR. Prevalência de barreiras para a prática de atividade física em adolescentes. Rev Bras Epidemiol 2010; 13: 94-104.

28. Bandura A. Health promotion by social cognitive means. Health Educ Behav 2004; 31:143-64.

29. Malta DC, Moura EC, Castro AM, Cruz DKA, Neto OLM, Monteiro CA. Padrão de atividade física em adultos brasileiros: resultados de um inquérito por entrevistas telefônicas. Epidemiol Serv Saúde 2009; 18:7-16.

30. Hallal PC, Bertoldi AD, Gonçalves H, Victora CG. Prevalência de sedentarismo e fatores associados em adolescentes de 10-12 anos de idade. Cad Saúde Pública 2006; 22:1277-87.

31. Kirby J, Levin KA, Inchley J. Parental and peer influences on physical activity among scottish adolescents: a longitudinal study. J Phys Act Health 2011; 8:785 -93.

32. Seabra AF, Mendonça DM, Thomis MA, Anjos LA, Maia JA. Determinantes biológicos e sócio-culturais associados à prática de atividade física de adolescentes. Cad Saúde Pública 2008; 24:721-36.

Recebido em 29/Jan/2013

Versão final reapresentada em 06/Jul/2013

Aprovado em 17/Out/2013 